\title{
Observational longitudinal study of symptom burden and time for recovery from community- acquired pneumonia reported by older adults surveyed nationwide using the CAP Burden of Illness Questionnaire
}

This article was published in the following Dove Press journal:

Patient Related Outcome Measures

30 July 2015

Number of times this article has been viewed

\section{Kathleen W Wyrwich' \\ Holly Yu \\ Reiko Sato ${ }^{2}$ \\ John H Powers ${ }^{3}$}

'Evidera, Inc., Bethesda, MD, USA; ${ }^{2}$ Pfizer Inc., Collegeville, PA, USA;

${ }^{3}$ George Washington University

School of Medicine and Health

Sciences, Washington, DC, USA
Correspondence: Kathleen WWyrwich Evidera, 7101 Wisconsin Avenue, Suite 600, Bethesda, MD 208I4, USA

$\mathrm{Tel}+\mathrm{I} 3 \mid 47738000$

Fax + I 3147738004

Email kathy.wyrwich@evidera.com
Background: Millions of older adults who develop community-acquired pneumonia (CAP) each year survive, but there is a large knowledge gap on the burden of CAP and the recovery process in survivors from the patient perspective.

Methods: The newly developed CAP Burden of Illness Questionnaire was administered through a Web survey to a nationwide sample of US adults aged $\geq 50$ years who were recently diagnosed with CAP. Survey respondents with unresolved symptoms or other CAP-related health problems completed a second survey 30 days later; a third survey was completed another 30 days later by respondents with unresolved symptoms or problems. Nationally representative results describing the average time to recovery of symptoms and other CAP-related problems were achieved using post-stratification weights.

Results: Five hundred participants completed the initial survey. The time to resolution for the CAP symptoms of weakness, shortness of breath, and tiredness exceeded 3 weeks on average. There was an average of 13 days of absenteeism, and 3 weeks (mean $=21$ days) before achieving full work/activity productivity after CAP. For participants with health conditions that worsened from pneumonia, chronic emphysema and chronic obstructive pulmonary disease took the longest to return to baseline (mean $=60$ and 52.4 days, respectively).

Conclusion: The results from this study demonstrate that older adults surviving a CAP episode experience a significant multi-symptom illness with long recovery periods to achieve pre-CAP health and productivity. These findings highlight the need for further research on effective clinician-patient communication, the need for patient-centered outcomes in clinical trials for CAP therapeutics, adequate home care during the recovery process, and the pursuit of CAP prevention strategies.

Keywords: burden of illness, community-acquired pneumonia, symptoms, patient-reported outcomes, longitudinal study, health-related quality of life

\section{Introduction}

Approximately, 950,000 cases of community-acquired pneumonia (CAP) occur in the USA each year in adults $<65$ years of age ${ }^{1}$ concurrently, another 1.3 million cases occur in older adults $\geq 65$ years of age. ${ }^{2} \mathrm{CAP}$ is defined as pneumonia contracted in the community rather than in a hospital or nursing home setting, or while on a ventilator. ${ }^{3}$ With the incidence and severity of CAP increasing notably with age, ${ }^{4}$ pneumonia remains the leading cause of infectious disease mortality in the USA. ${ }^{5}$ These data 
indicate that millions of older adults in the USA survive this disease each year, leaving a large knowledge gap on the burden of CAP and the recovery process in survivors from the patient perspective.

Prior studies evaluating the time to resolution of CAP symptoms have focused on a very limited number of indicators selected and worded by physicians. ${ }^{6,7}$ While returning to work or other usual activities are important milestones in the recovery from CAP, ${ }^{8-10}$ diminished productivity during the CAP recovery process may extend beyond absenteeism. There is a need to better understand the broad range of problems associated with CAP recovery based on the patients' experiences that are not measured in generic health status instruments. ${ }^{11,12}$

Therefore, the CAP Burden of Illness Questionnaire (CAP-BIQ) was developed and refined through in-depth concept elicitation and cognitive debriefing interviews with adults $\geq 50$ years of age who had recently been diagnosed with CAP. ${ }^{13}$ This report provides results across three successive monthly survey administrations up to 180 days after CAP diagnosis, detailing the length of time that older adults in the USA have CAP symptoms as well as CAP's detrimental effects on other health conditions and productivity.

\section{Methods}

\section{Study design, participants, and outcome measures}

The CAP-BIQ was designed using the methodology recommended by the US Food and Drug Administration ${ }^{14}$ for the development of patient-reported outcome measures. Details on the development and testing of this survey instrument are available elsewhere. ${ }^{13}$ The questionnaire was administered in three waves, each 30 days after the preceding wave (Wave 1, Wave 2, and Wave 3), to measure the time course of CAP symptoms until resolution. Participants with unresolved CAP symptoms or related CAP problems noted at Wave 1 were followed 30 days later (Wave 2); those with any unresolved CAP symptoms or problems at Wave 2 were followed for an additional 30 days (Wave 3 ). The full CAP-BIQ is available on the Journal's website at http://www.dovepress.com/ get supplementary file.php?f=85779.pdf.

Participants were recruited through an e-mail invitation from the YouGov ${ }^{\circledR}$ panel of US adults age $\geq 50$ years. For inclusion, participants were required to: have been told by a doctor or nurse that they had pneumonia within the past 120 days; self-report having a chest X-ray or computed tomography (CT) scan around the time they were informed of their diagnosis; be able to read English; and be willing to provide informed consent. To refine CAP identification, participants were excluded if they had been admitted to the hospital for any reason during the 2 weeks prior to their diagnosis or if they resided in a nursing home or assisted care facility. Enrollment was closed after the 500th participant completed the Wave 1 assessment; a sample size of 500 was chosen to give adequate precision on descriptive statistics. This study was conducted in accordance with the amended Declaration of Helsinki. An independent ethics and review committee (Ethical and Independent Review Services, Independence, MO, USA) approved the protocol, and written informed consent was obtained from all the participants.

\section{Statistical analyses}

Sample weighting was used to construct a nationally representative sample of the US adults aged $\geq 50$ years. The post-stratification weights were calculated using matching variables for age, race/ethnicity, and education. Student's $t$-tests were used to compare mean values, and chi-square tests for categorical data inferences. For time to resolution of CAP-related symptoms or other issues, the mean, median, and interquartile ranges, as well as $P$-values for the mean inferential comparisons were reported. As exploratory analyses, results were stratified to compare by: age group (50-64 and $\geq 65$ years old), hospitalization status (yes/no), and risk group assignment for developing pneumonia (high risk: the presence of asthma, COPD, chronic bronchitis, chronic emphysema, heart disease, diabetes, or other immunocompromised conditions; low risk: none of the high-risk comorbid conditions). ${ }^{15,16}$ Productivity and caregiver assistance results were stratified based on self-reported employment status. All the group comparison analyses incorporated a Bonferroni correction to alpha to address multiplicity. The analyses were performed in SAS version 9.1.3.

\section{Results}

The sociodemographic characteristics for all participants in the Wave 1 (baseline) survey using the weighted sample are fully described elsewhere. ${ }^{13}$ Briefly summarizing those results, $45 \%$ were male, the average age was 62.4 years old, the majority of participants reported living with a partner (79.5\%), and $89.2 \%$ selected White as their race. The mean number of days between CAP diagnosis and Wave 1 CAPBIQ completion was 56.9 days. The mean hospital stay due to pneumonia was 6.8 days, and $91.4 \%$ of these patients were hospitalized on the day of diagnosis. The hospitalized group was older and had a greater percentage of participants with COPD, chronic emphysema, heart disease, and diabetes than the nonhospitalized group $(P<0.001)$. High-risk participants 
were more likely to have high blood pressure, and more than twice as likely to be hospitalized compared to the low-risk group $(P<0.001){ }^{13}$

\section{Symptom resolution}

Length of time until symptom resolution for all participants who experienced at least 1 day with a symptom is reported in Table 1. The mean and median duration for fever (feeling warm), chills, and sweats was less than a week, whereas the mean time for tiredness, weakness, and shortness of breath were at least 3 weeks. The other ten symptoms measured in this study persisted generally for approximately 1-2 weeks. The interquartile ranges for cough, body aches, wheezing, and trouble sleeping show that, for at least a quarter of the sample, it took nearly 3 weeks to achieve symptom resolution (Table 1).

Only fever/warmth and chills differed significantly $(P \leq 0.001)$ between the age groups, with the younger group experiencing the symptoms on average approximately 1-3 days longer compared to the older group. Low- and highrisk groups had significant differences only in the duration of chills and sweats, with longer mean times (2-3 days) observed for the low-risk group. Fever lasted nearly twice as long on average in the nonhospitalized group compared to those who were hospitalized, but significant differences in mean symptom duration between these two groups indicated an overall longer recovery time for the hospitalized group for tiredness, body aches, and weakness.

\section{Time to resolution of health conditions worsened by pneumonia}

For those who experienced a worsening of specific health conditions due to pneumonia, the means, medians, and interquartile ranges for number of days to return to baseline are reported in Table 2. Patients with chronic emphysema had the longest duration to return to baseline $($ mean $/$ median $=60 / 65$ days) followed by COPD (52/44 days); the 75 th percentile levels for both of these groups were nearly 3 months (89 days). In the subgroup with COPD, the younger group took longer to return to their baseline COPD condition than the older age group $(P=0.004)$, as did hospitalized participants compared to nonhospitalized participants $(P=0.025)$, although these differences did not reach statistical significance when adjusted for multiplicity $(P<0.003)$.

\section{CAP impacts on productivity}

CAP impacts on work productivity and the need for additional help are presented in Table 3. There was a greater proportion of nonemployed versus employed participants who were hospitalized; however, a greater proportion of the employed group missed work (92.5\%) compared to the proportion of the nonemployed group who missed their usual daily activities $(74.1 \% ; P<0.001)$. Those who missed days of work or usual daily activities were away on average for 13 days. Moreover, the mean time before achieving full work/activities productivity took nearly 3 weeks (20.1 days), and differed between those who were employed (14.2 days) and those who were not $(24.3$ days; $P<0.001)$. As reported in Wyrwich et al (2013), 77.4\% of participants reported at Wave 1 on the need for help with their care from family or friends during their bout with CAP. ${ }^{13}$ On average, participants receiving this assistance had help for nearly 2 weeks (Table 3).

\section{Discussion}

This study evaluated the contemporary experience of the disease course of CAP in older adults in the USA by documenting the burden of illness in CAP survivors up to 180 days after diagnosis from the patient's perspective. Use of a newly developed, disease-specific measure based on what patients themselves described as important ${ }^{13}$ allowed greater detail on the presence, duration, and impact of CAP symptoms.

The key findings demonstrate that CAP patients experience a wide variety of systemic and respiratory symptoms that also impact the course of other comorbid illnesses. The results also show that symptoms and impacts of symptoms can last for days to months after onset of illness, imposing a burden on patients and on their caregivers and/or partners. Of the 16 symptoms measured, half took more than 2 weeks, on average, to resolve; the time to resolution for weakness, shortness of breath, and tiredness exceeded 3 weeks. There was also a delay of approximately 3 weeks in achieving full productivity at work or usual activities.

The length of time to resolution for most longer-lasting symptoms did not differ between participants in the younger and older age groups, or between the low- and high-risk groups. However, for tiredness, body aches, weakness, and trouble sleeping, resolution was nearly 1 week longer for those who were hospitalized compared to those who were not hospitalized. In contrast, early-resolving symptoms of fever/ warmth, sweats, or chills lasted longer in nonhospitalized or younger, and low-risk subgroups compared to their respective counterparts. Because CAP is caused by pathogenic organisms infecting the pulmonary parenchyma with resultant host inflammatory response, ${ }^{17,18}$ the relatively long duration of some symptoms may reflect prolonged host response that 


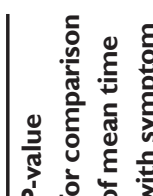

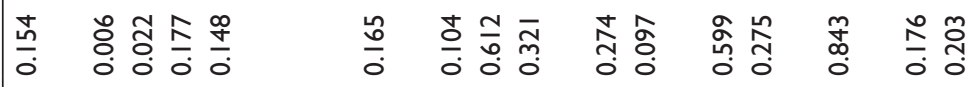

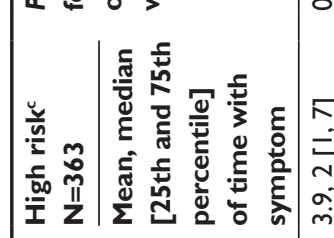

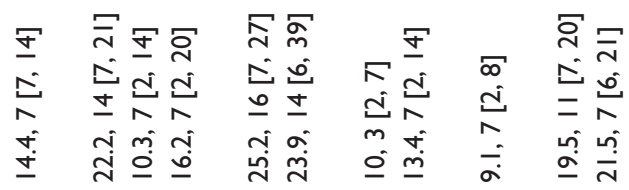

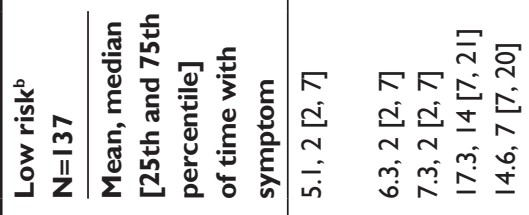

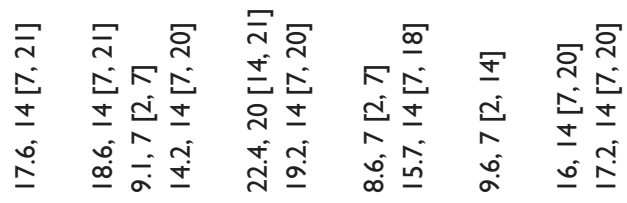

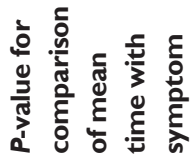

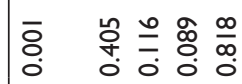

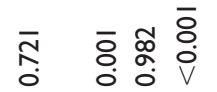

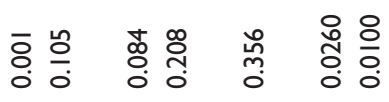

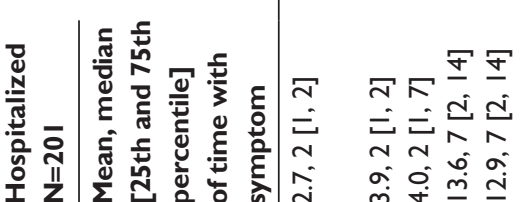

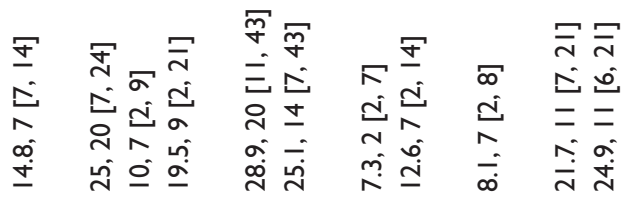

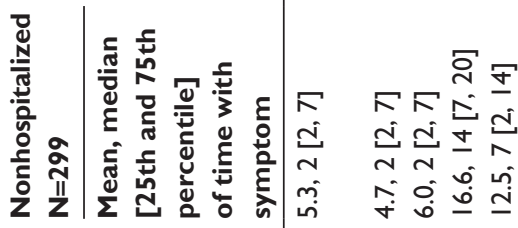

守守

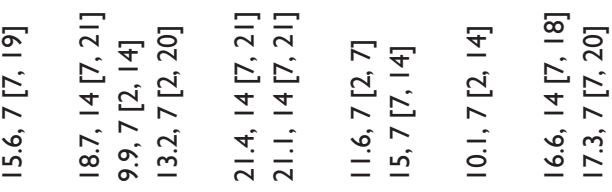

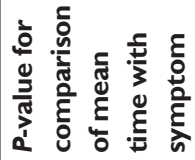

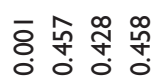

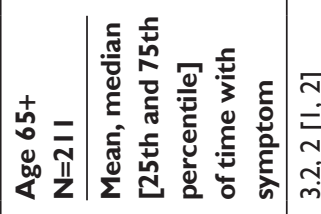

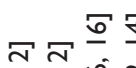

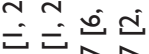

त) ते

นึ่

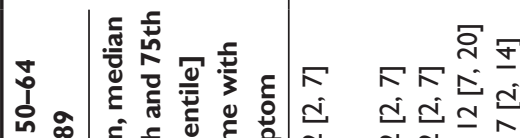

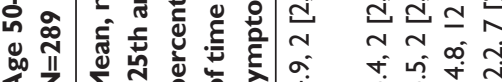

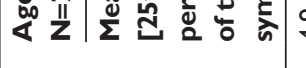

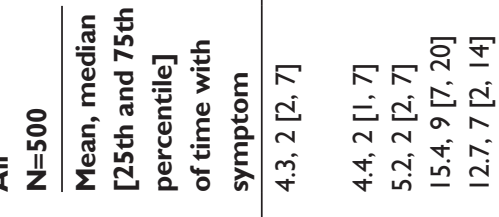

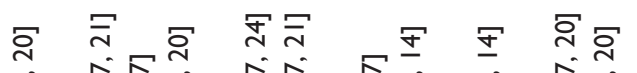

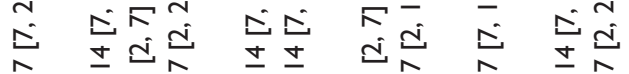

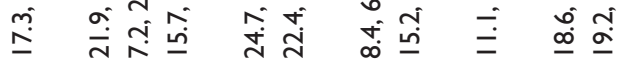




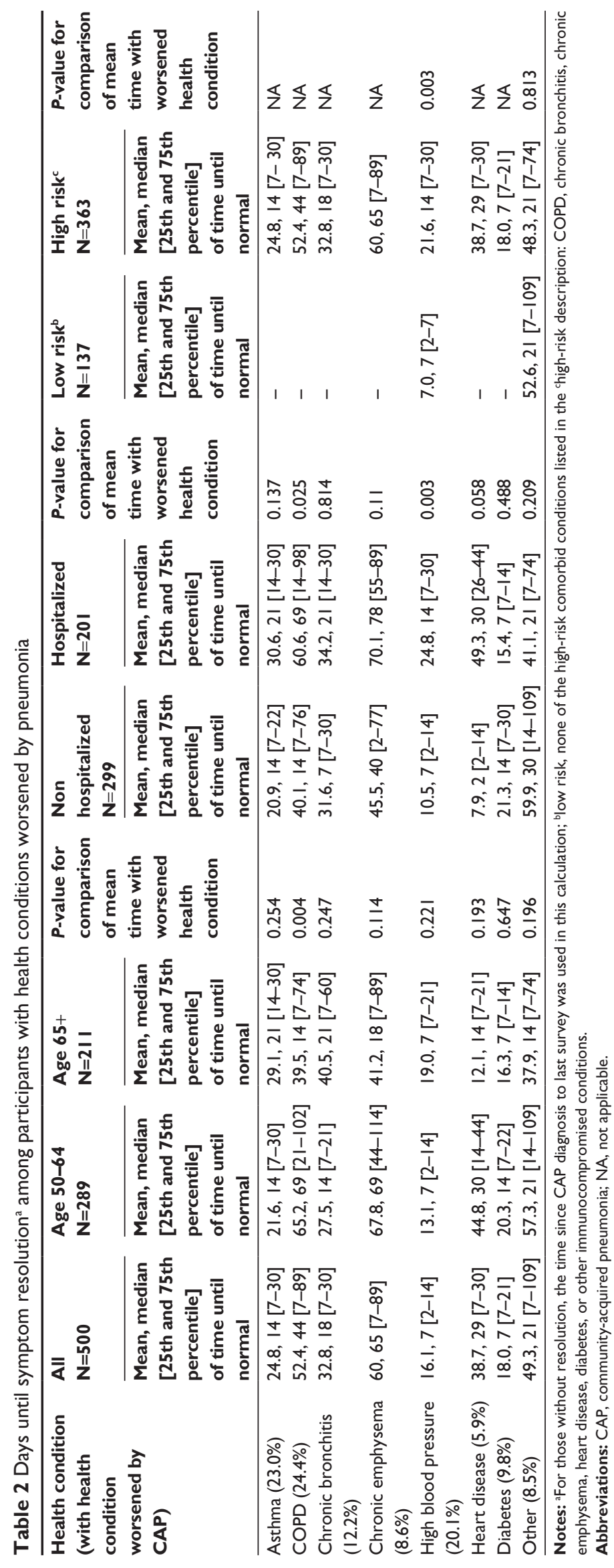


Table 3 Impact of community-acquired pneumonia on absenteeism, productivity, and help from caregiver by employment status

\begin{tabular}{|c|c|c|c|c|}
\hline Impacts on productivity and need for additional help & $\begin{array}{l}\text { All } \\
N=500\end{array}$ & $\begin{array}{l}\text { Employed } \\
\mathrm{N}=161\end{array}$ & $\begin{array}{l}\text { Not employed } \\
\mathrm{N}=339\end{array}$ & $\begin{array}{l}P \text {-value for } \\
\text { comparison }\end{array}$ \\
\hline$\%$ hospitalized & $40.29 \%$ & $29.98 \%$ & $45.19 \%$ & $0.00 I^{a}$ \\
\hline$\%$ who missed work or other usual daily activities & $79.99 \%$ & $92.48 \%$ & $74.06 \%$ & $<0.00 \mathrm{I}^{\mathrm{a}}$ \\
\hline $\begin{array}{l}\text { Number of days of work or other usual daily activities missed } \\
\text { due to pneumonia } \\
\text { Mean, median [ } 25 \text { th and } 75 \text { th percentile] }\end{array}$ & $12.9,8[5-14]$ & $9.8,7[4-14]$ & $14.7,10[6-15]$ & $<00 I^{\mathrm{b}}$ \\
\hline $\begin{array}{l}\text { Number of days until reached usual performance level in work } \\
\text { or other usual daily activities } \\
\text { Mean, median [25th and } 75 \text { th percentile] }\end{array}$ & $20.1, \mid 4[7-2 \mid]$ & $14.2,14[7-14]$ & $24.3,14[7-21]$ & $<0.00 \mathrm{I}^{\mathrm{b}}$ \\
\hline Percent with family or friends helping with care during pneumonia & $77.41 \%$ & $80.83 \%$ & $75.79 \%$ & $0.208^{\mathrm{a}}$ \\
\hline $\begin{array}{l}\text { Number of days of family or friends helping with care during pneumonia } \\
\text { Mean, median [25th and } 75 \text { th percentile] }\end{array}$ & $13.7,7[2-14]$ & $7.5,7[2-7]$ & $16.9,7[6-18]$ & $<0.00 \mathrm{I}^{\mathrm{b}}$ \\
\hline
\end{tabular}

Notes: ${ }^{a}$-value for percentage comparison; ${ }^{b}$-value for mean comparison.

is persistent even after immunological response and control of the causative organisms. The magnitude and duration of host response may differ in different types of patients, with or without antibiotics.

For participants who reported that CAP worsened their comorbid conditions, many of the symptoms of these conditions were affected for more than a month; younger or hospitalized participants with worsened COPD were generally affected for more than 2 months. Our results are consistent with prior investigations showing that over $35 \%$ of adults with CAP at low risk of death have unresolved CAP-related symptoms 1 month post-diagnosis. ${ }^{7}$

Our findings are also consistent with other studies showing: 1) older patients who survive CAP on average had similar symptoms but different severity or duration of symptoms than younger patients because of the former's diminished ability to mount a robust immune response, ${ }^{19,20}$ 2) CAP patients with other coexisting health conditions had significantly impaired general quality of life compared to CAP patients without underlying diseases ${ }^{21}$ and 3 ) a median time to return to work of 7 days for outpatients and 14 days for inpatients in low-risk CAP patients. ${ }^{8}$ Although time to return to full work/activity productivity has not been assessed in previous studies, the 3 weeks needed to return to full productivity even after return to work highlights the impact of CAP beyond traditional productivity measures.

This study has some important limitations. As in previous recovery studies, ${ }^{6,22}$ this study used retrospective recall of symptoms, which can be subject to recall bias. ${ }^{23,24}$ To recruit a sufficient sample of older adults with CAP, a window of up to 120 days between diagnosis and Wave 1 survey completion was necessary. To address the issue of recall bias, prospective studies on the burden of CAP in patients who are surveyed soon after diagnosis are needed to assess the duration of specific CAP symptoms and functioning. Such studies are currently underway based on the work in this study. ${ }^{25}$ The use of self-report for the diagnosis of pneumonia is also a limitation, although patients are likely to correctly remember a seminal event like the diagnosis of this potentially life-threatening illness. ${ }^{26,27} \mathrm{We}$ attempted to minimize case identification error by asking patients if they had a radiologically confirmed diagnosis - as radiography is another event that patients aged $\geq 50$ years are likely to accurately recall. ${ }^{28-30}$ Other studies have also used self-report of pneumonia diagnosis, ${ }^{31-33}$ one of which showed high concordance $(82 \%)$ between self-diagnosis and medical chart and radiographic confirmation of diagnosis. ${ }^{31}$ Moreover, if there was overdiagnosis of pneumonia in the current study because of contamination by patients with bronchitis or other self-resolving disease, the results would most likely underestimate the burden of symptoms. ${ }^{34}$ Another potential limitation that might bias the study results would be if the older adults with CAP in the YouGov ${ }^{\circledR}$ panel are different from the US general population for this age group in ways that cannot be fully adjusted for with the demographic weights that were used. Lastly, our study focused solely on CAP survivors and may not reflect the presence of symptoms at baseline in CAP patients who did not survive their bout with pneumonia. Therefore, there may be survivor bias in the comparisons of younger versus older patients and hospitalized versus nonhospitalized patients if older, hospitalized patients have greater mortality. Regardless, our results are accurate in terms of the clinical course of patients who will be seen in practice by clinicians, since they represent the population of survivors.

In addition, although CAP-BIQ captures data to provide a comprehensive picture of the course of illness and disease impact from the patient's perspective, this lengthy 
questionnaire may not be appropriate as an outcome measure in clinical trials testing treatment effects of various interventions for pneumonia. Nonetheless, the results from this patient-reported outcome questionnaire can inform the development of a shorter instrument for use in clinical trials measuring CAP impact at diagnosis and recovery. This work based on CAP-BIQ is the basis for ongoing studies to prospectively develop a patient-reported outcome for clinical trials in patients with radiographically confirmed CAP. ${ }^{25}$

Despite these limitations, this study offers many key insights into patients' experiences with CAP. A major strength of the study is the documentation of the content validity and understandability of the survey instrument before its use in this study, demonstrating a wide array of symptoms not captured in previous studies. ${ }^{13}$ Moreover, few studies have examined the long-term effects of CAP beyond the first month after diagnosis. Employing a broad Web panel provided for nationwide enrollment. The use of a single method of administration for the survey also eliminates potential bias due to a mixing of methods (eg, Web survey, paper and pencil, etc). In addition, knowledge of the participants' age, race/ethnicity, and education aided in the construction of post-stratification weights matching the US population aged $\geq 50$ years, and corrected for any demographic skewness in the sample.

With this evidence from a patient perspective on the range of CAP symptoms, functional limitations, and time necessary to fully recover, our results can have an impact on clinical practice. Greater understanding of the natural history of CAP can aid both patients and clinicians in better managing episodes beyond the acute phase of the disease. Our data show a broader range of symptoms than cough, chest pain, and dyspnea, which are usually associated with pneumonia, indicating that clinicians should inquire about a broader range of symptoms. Symptoms often persist longer than a month, which exceeds the time when patients complete antibiotic therapy. Therefore, patients should be informed that they may continue to experience the residual effects of pneumonia long beyond the period of active treatment. Patient knowledge of the course of the treated disease allows for realistic expectations of the recovery trajectory, and may reduce unnecessary health care use and inappropriate antibiotic prescribing for apparent relapses that actually represent the natural course of the illness. A previous study reported greater pneumoniarelated ambulatory care visits ${ }^{6}$ with greater symptom burden during CAP recovery, independent of the pneumonia severity or comorbidities.
These results also demonstrate that adequate home care for a sufficient period is a key consideration, one that needs to be addressed either at the time of diagnosis (outpatient recovery) or during hospitalization prior to discharge. The seriousness and disability associated with CAP over an extended period is evidenced by $77 \%$ of the study population receiving informal care from family or friends for almost 2 weeks. Given that 30-day pneumonia readmission rate is tracked as an indicator of quality and coordination of care by the Center for Medicare and Medicaid Services, ${ }^{35}$ providers should involve caregivers in the post-discharge planning and follow-up to reduce ambulatory care-sensitive readmissions.

Our results also provide evidence to inform future research efforts. Future studies may evaluate whether some treatments have greater effects than others in decreasing various symptoms associated with CAP. Current studies focus solely on resolution of a small number of early-resolving symptoms (eg, chest pain, cough, and dyspnea); yet the demand for patient-centered outcomes fully reflecting the patient's experience has become a major impetus in health care reform. ${ }^{36}$ Therefore, description of treatment effects on the spectrum of important CAP symptoms deserves further attention. Our results show a wider range of symptoms in pneumonia than are usually captured in clinician-reported outcomes in the clinical trials of CAP.

Finally, in addition to the substantial number of CAP deaths annually, this documented burden of illness among CAP survivors emphasizes the importance of CAP prevention in older adults to avoid the prolonged disability associated with this disease. Recommended prevention strategies include smoking cessation, annual influenza vaccination, pneumococcal vaccine for adults aged $\geq 65$ years and those $<65$ years old who have underlying medical conditions, and educational support for these vulnerable populations. $^{37}$

\section{Conclusion}

This study demonstrated that older adults surviving a CAP episode experience a significant multi-symptom illness with long recovery period before achieving pre-CAP health and productivity. These findings highlight the importance of communicating the natural course of the illness to inform patient and clinician expectations, the need for further research on effective clinician-patient communication, adequate home care during the recovery process, better patient-centered outcomes in evaluating therapies for CAP, and the pursuit of CAP prevention strategies. 


\section{Acknowledgments}

The authors acknowledge the valuable assistance of Elizabeth Nicole Bush in the development and execution of this study, and Anna Steenrod in the development of this manuscript as paid employees of Evidera (formerly a division of United BioSource Corporation). This study was sponsored by Wyeth, which was acquired by Pfizer Inc. in October 2009.

\section{Author contributions}

KWW had full access and control to all the data in the study and takes responsibility for the integrity of the data and the accuracy of the data analysis. KWW also takes responsibility for the content of the manuscript, including the data and analysis. All four authors participated in the study design, data collection process, and analyses and interpretation, and agree to allow the journal to review their data if requested. All four authors participated in the manuscript writing, review, and approval to submit this paper to Patient Related Outcome Measures. Written permission was obtained from all corresponding authors prior to submission.

\section{Disclosure}

$\mathrm{HY}$ and RS are employees of Pfizer Inc. KWW is an employee of Evidera, Inc., formerly a division of United BioSource Corporation, which received financial support from Pfizer in connection with the study development and execution as well as the manuscript development. JHP was a paid clinical consultant to Pfizer in connection with the development, execution, and interpretation of the study results but not in the development or writing of the manuscript. The authors report no other conflicts of interest in this work.

\section{References}

1. Bonafede MM, Suaya JA, Wilson KL, Mannino DM, Polsky D. Incidence and cost of CAP in a large working-age population. Am J Manag Care. 2012;18(7):380-387.

2. Yu H, Rubin J, Dunning S, Li S, Sato R. Clinical and economic burden of community-acquired pneumonia in the Medicare fee-for-service population. J Am Geriatr Soc. 2012;60(11):2137-2143.

3. National Center for Health Statistics. Health, United States, 2006. Hyattsville, MD: US Department of Health and Human Services; 2006.

4. File TM Jr, Marrie TJ. Burden of community-acquired pneumonia in North American adults. Postgrad Med. 2010;122(2):130-141.

5. Heron MP, Hoyert DL, Xu J, Scott C, Tejada-Vera B. Deaths: preliminary data for 2006. Natl Vital Stat Rep. 2008;56(16):1-52.

6. Metlay JP, Fine MJ, Schulz R, et al. Measuring symptomatic and functional recovery in patients with community-acquired pneumonia. J Gen Intern Med. 1997;12(7):423-430.

7. Metlay JP, Atlas SJ, Borowsky LH, Singer DE. Time course of symptom resolution in patients with community-acquired pneumonia. Respir Med. 1998;92(9):1137-1142.
8. Labarere J, Stone RA, Obrosky DS, et al. Comparison of outcomes for low-risk outpatients and inpatients with pneumonia: a propensityadjusted analysis. Chest. 2007;131(2):480-488.

9. Fine MJ, Stone RA, Singer DE, et al. Processes and outcomes of care for patients with community-acquired pneumonia: results from the Pneumonia Patient Outcomes Research Team (PORT) cohort study. Arch Intern Med. 1999;159(9):970-980.

10. Brandenburg JA, Marrie TJ, Coley CM, et al. Clinical presentation, processes and outcomes of care for patients with pneumococcal pneumonia. J Gen Intern Med. 2000;15(9):638-646.

11. El Solh A, Pineda L, Bouquin P, Mankowski C. Determinants of short and long term functional recovery after hospitalization for communityacquired pneumonia in the elderly: role of inflammatory markers. $B M C$ Geriatr. 2006;6:12.

12. Marrie TJ, Lau CY, Wheeler SL, Wong CJ, Vandervoort MK, Feagan BG. A controlled trial of a critical pathway for treatment of community-acquired pneumonia. CAPITAL Study Investigators. Community-Acquired Pneumonia Intervention Trial Assessing Levofloxacin. JAMA. 2000;283(6):749-755.

13. Wyrwich KW, Yu H, Sato R, Strutton D, Powers JH. Communityacquired pneumonia: symptoms and burden of illness at diagnosis among US adults aged 50 years and older. Patient. 2013;6(2):125-134.

14. US Food and Drug Administration. Guidance for industry on patientreported outcome measures: use in medical product development to support labeling claims. Fed Regist. 2009;74(235):65132-65133.

15. Centers for Disease Control and Prevention. Prevention of pneumococcal disease: recommendations of the Advisory Committee on Immunization Practices (ACIP). MMWR Recomm Rep. 1997;46(RR-8):1-24.

16. Centers for Disease Control and Prevention. Updated recommendations for prevention of invasive pneumococcal disease among adults using the 23-valent pneumococcal polysaccharide vaccine (PPSV23). MMWR Morb Mortal Wkly Rep. 2010;59(34):1102-1106.

17. Calbo E, Alsina M, Rodriguez-Carballeira M, Lite J, Garau J. The impact of time on the systemic inflammatory response in pneumococcal pneumonia. Eur Respir J. 2010;35(3):614-618.

18. Kellum JA, Kong L, Fink MP, et al. Understanding the inflammatory cytokine response in pneumonia and sepsis: results of the Genetic and Inflammatory Markers of Sepsis (GenIMS) Study. Arch Intern Med. 2007;167(15):1655-1663.

19. Metlay JP, Schulz R, Li YH, et al. Influence of age on symptoms at presentation in patients with community-acquired pneumonia. Arch Intern Med. 1997;157(13):1453-1459.

20. Fernandez-Sabe N, Carratala J, Roson B, et al. Community-acquired pneumonia in very elderly patients: causative organisms, clinical characteristics, and outcomes. Medicine (Baltimore). 2003;82(3): 159-169.

21. Schnoor M, Schoefer Y, Henrich G, Raspe H, Schaefer T. General and health-related life satisfaction of patients with community-acquired pneumonia. Psychol Health Med. 2009;14(3):331-342.

22. El Moussaoui R, Opmeer BC, de Borgie CA, et al. Long-term symptom recovery and health-related quality of life in patients with mild-tomoderate-severe community-acquired pneumonia. Chest. 2006;130(4): 1165-1172.

23. Norman GR, Stratford P, Regehr G. Methodological problems in the retrospective computation of responsiveness to change: the lesson of Cronbach. J Clin Epidemiol. 1997;50(8):869-879.

24. Ross M. Relation of implicit theories to the construction of personal histories. Psychol Rev. 1989;96:341-357.

25. Howard K, Portalupi S, Hoffmann S, et al. Development of a new patient reported outcome (PRO) measure for community-acquired bacterial pneumonia (CABP). Value Health. 2014;17(3):A280.

26. D'Argembeau A, Comblain C, van der Linden M. Phenomenal characteristics of autobiographical memories for positive, negative, and neutral events. Appl Cogn Psychol. 2003;17:281-294.

27. Talarico JM, LaBar KS, Rubin DC. Emotional intensity predicts autobiographical memory experience. Mem Cognit. 2004;32(7):1118-1132. 
28. Bergmann MM, Byers T, Freedman DS, Mokdad A. Validity of selfreported diagnoses leading to hospitalization: a comparison of selfreports with hospital records in a prospective study of American adults. Am J Epidemiol. 1998;147(10):969-977.

29. Skull SA, Andrews RM, Byrnes GB, et al. Validity of self-reported influenza and pneumococcal vaccination status among a cohort of hospitalized elderly inpatients. Vaccine. 2007;25(25):4775-4783.

30. Bush TL, Miller SR, Golden AL, Hale WE. Self-report and medical record report agreement of selected medical conditions in the elderly. Am J Public Health. 1989;79(11):1554-1556.

31. Baik I, Curhan GC, Rimm EB, Bendich A, Willett WC, Fawzi WW. A prospective study of age and lifestyle factors in relation to community-acquired pneumonia in US men and women. Arch Intern Med. 2000;160(20):3082-3088.

32. Neuman MI, Willett WC, Curhan GC. Vitamin and micronutrient intake and the risk of community-acquired pneumonia in US women. Am J Med. 2007;120(4):330-336.

33. Neuman MI, Willett WC, Curhan GC. Physical activity and the risk of community-acquired pneumonia in US women. Am J Med. 2010;123(3): 281.e7-281.e11.
34. Little P, Stuart B, Moore M, et al. Amoxicillin for acute lowerrespiratory-tract infection in primary care when pneumonia is not suspected: a 12-country, randomised, placebo-controlled trial. Lancet Infect Dis. 2013;13(2):123-129.

35. Center for Medicare and Medicaid Services. Outcome Measures. Available from: http://www.cms.gov/Medicare/Quality-Initiatives-PatientAssessment-Instruments/HospitalQualityInits/OutcomeMeasures.html. Accessed April 1, 2013.

36. Patient-Centered Outcome Research Institute. PCORI methodology standards; 2012. Available from: http://www.pcori.org/assets/PCORIMethodology-Standards.pdf. Accessed March 4, 2013.

37. Mandell LA, Wunderink RG, Anzueto A, et al. Infectious Diseases Society of America/American Thoracic Society consensus guidelines on the management of community-acquired pneumonia in adults. Clin Infect Dis. 2007;44(Suppl 2):S27-S72.
Patient Related Outcome Measures

\section{Publish your work in this journal}

Patient Related Outcome Measures is an international, peer-reviewed, open access journal focusing on treatment outcomes specifically relevant to patients. All aspects of patient care are addressed within the journal and practitioners from all disciplines are invited to submit their work as well as healthcare researchers and patient support groups.

\section{Dovepress}

The manuscript management system is completely online and includes a very quick and fair peer-review system. Visit http://www.dovepress. com/testimonials.php to read real quotes from published authors.

Submit your manuscript here: http://www.dovepress.com/patient-related-outcome-measures-journal 